\title{
Generalised Inverse Scattering for a Linear PDE Associate to $\mathrm{KdV}$
}

\author{
P C SABATIER \\ Laboratoire de Physique Mathématique et Théorique Université des Sciences et \\ Techniques du Languedoc, Place Eugǹe Bataillon \\ 34095 - MONTPELLIER (France) Cedex 05. \\ E-mail : sabatier@lpta.univ-montp2.fr
}

This article is part of the special issue published in honour of Francesco Calogero on the occasion of his 70th birthday

\begin{abstract}
Inverse Scattering methods for solving integrable nonlinear p.d.e. found their limits as soon as one tried to solve with them new boundary value problems. However, some of these problems, e.g. the quarter-plane problem, can be solved (e.g. by Fokas linear methods), for related linear p.d.e., (e.g. LKdV). It is shown here that a nonlinear algebraic inverse scattering method, which we already applied to nonlinear KdV, but with only partial results, gives the full solution of the quarter-plane problem of another linear p.d.e. associated to KdV. The method makes use of generalised Lax equations and their solutions.
\end{abstract}

\section{Introduction}

It is well known that the "Inverse Scattering Method" solves ${ }^{(1)}$ nonlinear integrable p.d.e. When the boundary value condition is given on an axis where it enables one to calculate scattering coefficients by solving the "direct scattering problem", whereas, after translating the axis, the coefficients can be recalculated, the method yields the p.d.e. solution by solving the "inverse scattering problem". Attempts to generalize it to other boundary value problems were never really successful. Yet, we showed ${ }^{(2)}$ that several integrable nonlinear p.d.e. are associated to a generalisation of scattering called "global scattering" in the $x, t$ plane where Lax equations are the scattering equations such that enforcing both of them reduces the solutions to a two-dimensional set. As a consequence, it enables us to make a generalisation of Potential Scattering whose several mathematical tools reappear. Inverse Scattering Method is a quite trivial consequence of this "global scattering" process. However, we were not able to use it for solving the quarter-plane boundary value problem of $\mathrm{KdV}$, defined by equations (1.1) and (1.2) :

$$
\frac{\partial V}{\partial t}+\frac{1}{4} V^{\prime \prime \prime}-\frac{3}{2} V V^{\prime}=0
$$


where "prime" denotes $x$-derivatives,

Data : $V(x, 0), V(0, t), x \geqslant 0, t \geqslant 0$.

On the other hand, starting also from Lax equations, linear Fourier methods used by Fokas $^{(3)}$ gave a full solution to the quarter-plane boundary value problem of LKdV, i.e. (1.2) and

$$
\frac{\partial V}{\partial t}+\frac{1}{4} V^{\prime \prime \prime}=0
$$

Using a generalisation of Fokas method into the framework of global scattering we obtained a method that applies to (1.1), (1.2), but is not satisfactory outside of a poorly identified iteration range.

The global scattering process does not hold in the case of linearised KdV equations because what we call the corresponding Lax equations are not homogeneous. However, part of its results can be derived (we did it ${ }^{(2)}$ for $\mathrm{LKdV}$ ) if we reformulate the (consistency) relation between Lax equations that is equivalent to the (linear or nonlinear) p.d.e. we study. Such reformulations will be generalised in the present paper and then applied to yield full solutions of another linear p.d.e. associated to KdV :

$$
\frac{\partial a}{\partial t}+\frac{1}{4} a^{\prime \prime \prime}-\frac{3}{4}\left(a V^{\prime}+a^{\prime} V\right)=0
$$

where $V(x, t)$ is now a given solution of $\mathrm{KdV}, a(x, t)$ the solution of (1.4) we are seeking and the boundary condition may be either a one axis condition or condition (1.2). Of course, $V$ itself is a special solution of Eq. (1.4), but, a priori, with different boundary conditions.

\section{Correspondence between the p.d.e. and its "Lax" equations}

In previous papers $(2,4)$ we wrote down the Lax equations for KdV or for NLS as a system of two linear first order p.d.e. (variables $x$ and $t$ ) which have a common solution such that its second order cross derivatives be invariant under the $(x, t)$ permutation (the so-called consistency condition). Here, for any function $F$ which has second order derivatives, we define its " $\delta^{2}-$ shift" as

$$
\delta^{2} / F=: \frac{\partial^{2} F}{\partial t \partial x}-\frac{\partial^{2} F}{\partial x \partial t}
$$

The condition $\delta^{2} / F=0$, called "consistency condition", is very important. If we try to construct $F$ from either $\frac{\partial F}{\partial t}$ or $\frac{\partial F}{\partial x}$, and its value at, say, $\left(x_{0}, t_{0}\right)$, as it can be done from equations below, the various possible integral equations give the same $F$ if the consistency 
condition is fulfilled ; they are then equivalent to one integral equation where the crossderivatives common value is integrated over a rectangle as $\left(x_{0}, t_{0}\right) \cdot\left(x, t_{0}\right) \cdot(x, t) \cdot\left(x_{0}, t\right)$.

We shall be interested by three systems of two p.d.e. for two-vector functions:

$$
\begin{aligned}
& \frac{\partial E}{\partial x}=\mathbf{M}_{0} E ; \frac{\partial E}{\partial t}=\mathbf{N}_{0} E \\
& \frac{\partial G}{\partial x}=\left(\mathbf{M}_{0}+\mathbf{V}\right) G ; \frac{\partial G}{\partial t}=\left[\mathbf{N}_{0}+\mathbf{W}\right] G \\
& \frac{\partial \Phi}{\partial x}=\mathbf{M}_{0} \Phi+\mathbf{A} G ; \frac{\partial \Phi}{\partial t}=\mathbf{N}_{0} \Phi+\mathbf{B} G
\end{aligned}
$$

where

$$
\begin{aligned}
& \mathbf{M}_{0}=\left(\begin{array}{cc}
0 & 1 \\
-k^{2} & 0
\end{array}\right) \quad ; \quad \mathbf{N}_{0}=k^{2} \mathbf{M}_{0} \\
& \mathbf{V}=\left(\begin{array}{cc}
0 & 0 \\
V & 0
\end{array}\right) \quad ; \quad \mathbf{W}=\left(\begin{array}{cc}
V_{1} & V_{0} \\
k^{2} V_{0}+V_{2} & -V_{1}
\end{array}\right) \\
& \mathbf{A}=\left(\begin{array}{ll}
0 & 0 \\
a & 0
\end{array}\right) \quad ; \quad \mathbf{B}=\left(\begin{array}{cc}
a_{1} & a_{0} \\
b & -a_{1}
\end{array}\right) \\
& E=\left(\begin{array}{c}
e \\
e^{\prime}
\end{array}\right) ; \quad G=\left(\begin{array}{c}
g \\
g^{\prime}
\end{array}\right) ; \Phi=\left(\begin{array}{c}
\varphi \\
\varphi^{\prime}
\end{array}\right)
\end{aligned}
$$

Assumption A

$V(x, t)$ has at least a first order time-derivative and third order $x$ derivative.

We set

$$
V_{0}=\frac{1}{2} V, V_{1}=-\frac{1}{4} V^{\prime}, V_{2}=-\frac{1}{4} V^{\prime \prime}+\frac{1}{2} V^{2}
$$

and we extend these assumptions and notations to $a(x, t)$.

Now, it is easy to see that $E$ is a linear combination of $E^{+}$and $E^{-}$, with coefficients depending neither on $x$ or $t$ :

$$
E^{ \pm}(k, x, t)=\left(\begin{array}{c}
1 \\
\pm i k
\end{array}\right) \exp \left[ \pm i\left(k x+k^{3} t\right)\right]
$$

The $\delta^{2}$-shift of $E^{+}$, or $E^{-}$, vanishes. That of $G$ is 


$$
\delta^{2} / G=\left(\frac{0}{V g}\right) ; \bar{V}=\frac{\partial V}{\partial t}+\frac{1}{4} V^{\prime \prime \prime}-\frac{3}{2} V V^{\prime}
$$

In (2.11), we rediscover ${ }^{(4)}$ that (2.3) is a set of Lax equations enforcing $\mathrm{KdV}$ if $\delta^{2} / G=0$, which is assumed hereafter :

$$
\delta^{2} / G=\bar{V}=0
$$

As a matter of fact, $G(k, x, t)$ can be calculated at fixed $x$ or at fixed $t$ by solving in each case the corresponding equation (2.3), with a function $V(x, t)$ satisfying Assumption A which is a solution of $\mathrm{KdV}$ (the equations are readily transformed ${ }^{(4)}$ into linear Volterra equations).

Contrarily to Eqs (2.2) and (2.3), the equations (2.4) are not homogeneous, so that we cannot apply the zero trace theorem as we did it ${ }^{(2.4)}$ previously to settle the "global scattering" process. Setting $b$ equal to $a_{0} V+a_{1}^{\prime}+k^{2} a_{0}$, we easily obtain

$$
\delta^{2} / \Phi=\left(\begin{array}{c}
0 \\
\bar{a} g
\end{array}\right) ; \bar{a}=\frac{\partial a}{\partial t}+\frac{1}{4} a^{\prime \prime \prime}-\frac{3}{4}(a V)^{\prime}
$$

Hence the equation (1.4) is implied by the consistency condition $(\bar{a}=0)$ for $\Phi$. For $V=0,(2.3)$ reduces to $(2.2)$, and $(2.4)$ reduces to the equation we previously ${ }^{(2)}$ introduced to study LKdV, $a(x, t)$ being therefore a solution of (1.3).

In this generalisation of our previous approach, we shall calculate $\delta^{2}$-shifts of the determinants made of two solutions of the equations (2.2), (2.3), (2.4). In the "global scattering" process, these determinants had the physical meaning of scattering coefficients. Here we shall not seek a physical meaning in a global scattering process but only some relations with scattering coefficients on fixed $t$ axes.

Useful identities

Let $X$ and $Y$ be two 2-vectors (columns),

$$
\operatorname{det}(X, Y)=\widetilde{X} \tau Y
$$

where $\sim$ stands here for the transposition, which takes a column vector into a line one, and $\tau$ is the matrix $\left(\begin{array}{cc}0 & 1 \\ -1 & 0\end{array}\right)$.

One easily checks :

$$
\tau^{2}=-\left(\begin{array}{cc}
1 & 0 \\
0 & 1
\end{array}\right) \quad ; \quad \tau\left(\begin{array}{cc}
\alpha & \beta \\
\gamma & -\alpha
\end{array}\right) \tau=\left(\begin{array}{cc}
\alpha & \gamma \\
\beta & -\alpha
\end{array}\right)
$$

Note that the transposition effect shown in (2.15) holds only if the matrix is zero-trace. If the vectors $X$ and $Y$ are functions of $x$ and $t$, Eqs (2.14) and (2.15) yield 


$$
\delta^{2} / \operatorname{det}(X, Y)=\left(\delta^{2} / \tilde{X}\right) \tau Y+\widetilde{X} \tau\left(\delta^{2} / Y\right)
$$

and for $E, G$, and $\Phi$, using $(2.2),(2.3)$ and (2.4)

$$
\begin{aligned}
& \frac{\partial^{2}}{\partial t \partial x}\left(\widetilde{G}^{ \pm} \tau \Phi\right)=\frac{\partial}{\partial t}\left[\widetilde{G}^{ \pm} \tau(\mathbf{A} G-\mathbf{V} \Phi)\right]=\frac{\partial}{\partial x}\left[\widetilde{G}^{ \pm} \tau(\mathbf{B} G-\mathbf{W} \Phi)\right]+\delta^{2} / \widetilde{G}^{ \pm} \tau \Phi \\
& \frac{\partial^{2}}{\partial t \partial x}\left(\widetilde{E}^{ \pm} \tau \Phi\right)=\frac{\partial}{\partial t}\left[\widetilde{E}^{ \pm} \tau \mathbf{A} G\right]=\frac{\partial}{\partial x}\left[\widetilde{E}^{ \pm} \tau \mathbf{B} G\right]+\widetilde{E}^{ \pm} \tau \delta^{2} / \Phi \\
& \frac{\partial^{2}}{\partial t \partial x}\left(\widetilde{E}^{ \pm} \tau G\right)=\frac{\partial}{\partial t}\left[\widetilde{E}^{ \pm} \tau \mathbf{V} G\right]=\frac{\partial}{\partial x}\left[\widetilde{E}^{ \pm} \tau \mathbf{W} G\right]+\widetilde{E}^{ \pm} \tau \delta^{2} / G
\end{aligned}
$$

So as to give a precise definition of $G^{ \pm}$, and use scattering techniques, we impose Assumption $B$

$V$, $a$, and the derivatives cited in Assumption A vanish rapidly enough at $x \rightarrow \infty$, this meaning that

(a) we assume it as $x \rightarrow \pm \infty$ if we work on a full $x$-axis, as $x \rightarrow+\infty$ if we work on the $x>0$ half axis.

(b) $G$ and $\Phi$ are asymptotic to linear combinations of $E^{+}$and $E^{-}$: we call $G^{+}(k, x, t)$ the solution $G$ of $(2.3)$ which is asymptotic to $E^{+}(k, x, t)$ as $x \rightarrow+\infty$, and (if necessary) $G^{-}(k, x, t)$ that which is asymptotic to $E^{-}(k, x, t)$ as $x \rightarrow-\infty . G^{ \pm}$can be constructed ${ }^{(4)}$ from $V$ and $E^{ \pm}$by solving a convenient linear Volterra equation ${ }^{(2)}$.

\section{Boundary value condition on a full axis}

Assume we know $a(x, 0)$ for all $x \in \mathbb{R}$, and we are given $V(x, t)$ for all $x \in \mathbb{R}$, all $t>0$ finite, both going to zero, with derivatives, as $x \rightarrow \pm \infty$, fast enough for our assumptions. We seek a solution of (1.4) reducing to $a(x, 0)$ for $t=0$. We use equations $(2.3),(2.4)$, and choose in this section $G=G^{+}(k, x, t)$. We must enforce $\delta^{2} / \Phi=0$ and if we do it, $a$ satisfies (1.4). One can see from (2.16) that it is done if $\operatorname{det}\left[E^{+}, \Phi\right]$ and $\operatorname{det}\left[E^{-}, \Phi\right]$ have both their $\delta^{2}$-shift equal to zero, so that, according to (2.18)

$$
\frac{\partial}{\partial t}\left[\widetilde{E}^{ \pm} \tau A G\right]=\frac{\partial}{\partial x}\left[\widetilde{E}^{ \pm} \tau B G\right]
$$

Integrating along $\mathbb{R}$ yields

$$
\frac{\partial}{\partial t}\left[\int_{-\infty}^{+\infty} a(x, t) e^{ \pm i\left(k x+k^{3} t\right)} g^{+}(k, x, t) d x\right]=0
$$

or 


$$
\begin{aligned}
& \int_{-\infty}^{+\infty} a(x, t) e^{ \pm i\left(k x+k^{3} t\right)} g^{+}(k, x, t) d x \\
& =\int_{-\infty}^{+\infty} a(x, 0) e^{ \pm i k x} g^{+}(k, x, 0) d x
\end{aligned}
$$

For $V=0, a$ solves LKdV, and is readily obtained from (3.3) because the integral with $e^{+}$is the Fourier transform $\widetilde{a}(k, t)$ times $e^{2 i k^{3} t}$. One may notice on the way that $\widetilde{a}$ is the Born approximation of a reflection coefficient. Inversing the Fourier transform yields $a(x, t)$ from $\widetilde{a}$ and (3.3) is an evolution scheme quite similar to that known in the inverse scattering treatment of $\mathrm{KdV}$. These remarks hold for $V \neq 0$ except that the Fourier transform is replaced by an expansion over products $e^{+}(k, x, t) g^{+}(k, x, t)$. Of course, $e^{-i k^{3} t} g^{+}(k, x, t)$ is the Jost solution of the Schrödinger equation obtained from the first equation (2.3) and is known, since $V(x, t)$ is given. The products $e^{-2 i k^{3} t} e^{+}(k, x, t) g^{+}(k, x, t)$, given for $k \in \mathbb{R}$ and for the discrete spectrum $k=i \kappa_{1}, i \kappa_{2}, \cdots i \kappa_{N}$ of the Schrödinger operator, have completeness properties. There even exists an exact inversion formula, which is simple only in the case without bound state : $f^{+}(k, x, t)$ being the Jost solution asymptotic to $e^{i k x}$ as $x \rightarrow \infty$ (i.e. $e^{-i k^{3} t} g^{+}(k, x, t)$, and $f^{-}(k, x, t)$ the one asymptotic to $e^{-i k x}$ as $x \rightarrow-\infty$, $T(k)$ the transmission coefficient of the scattering problem by $V(x, t)$ on the $x$ line at fixed $t$, for any $h(x) \in L^{1}(\mathbb{R})$, we can write ${ }^{(5)}$ down if there is no bound state :

$$
\int_{-\infty}^{x} h(y) d y=-\frac{1}{2 \pi i} \int_{-\infty}^{+\infty} d k \frac{T(k)}{k} e^{-i k x} f^{-}(k, x, t) \int_{-\infty}^{+\infty} e^{i k y} f^{+}(k, y, t) h(y) d y
$$

and since we "know" $T, f^{-}, f^{+}$, Eq. (3.3) is readily solved in favor of $a(x, t)$. As a matter of fact, $T, f^{-}, f^{+}$, have to be calculated first so that a more indirect method, which we state below, is not much heavier and works as well if there are bound states :

On the $x$ line at fixed $t$, we first construct ${ }^{(6)}$ the transformation kernel $\kappa(x, y, t)$ corresponding to $V(x, t)$. This construction is equivalent to that of $f^{+}(k, x, t)$ which is given ${ }^{(6)}$ by

$$
f^{+}(k, x, t)=e^{i k x}+\int_{x}^{\infty} \kappa(x, y, t) e^{i k y} d y
$$

Notice by the way that (3.5) reminds us that $f^{+}$is holomorphic for $\operatorname{Im} k>0$, so that (3.3) extends to this domain. Eq. (3.3) shows the time invariance of $e^{(1 \pm 1) i k^{3} t} s^{ \pm}(k, t)$, where

$$
s^{ \pm}(k, t)=\int_{-\infty}^{+\infty} a(x, t) e^{ \pm i k x} f^{+}(k, x, t) d x
$$

By the way, notice that $s^{+}$is analogous to the reflection coefficient of the Inverse Scattering Method, and $s^{-}$to the transmission coefficient. So as to go further with $s^{+}$, we calculate its Fourier transform then substitute (3.5) in (3.6): 


$$
\begin{aligned}
& \widetilde{a}(z, t)=\pi^{-1} \int_{-\infty}^{+\infty} s^{+}(k, t) e^{-2 i k z} d k \\
& \widetilde{a}(z, t)=a(z, t)+\int_{-\infty}^{z} d x \kappa(x, 2 z-x, t) a(x, t)
\end{aligned}
$$

$s^{+}(k, 0)$ is given from the boundary value condition and $s^{+}(k, t)$ is $e^{-2 i k^{3} t} s^{+}(k, 0)$ so that $\widetilde{a}(z, t)$ is derived by (3.7), and $a(z, t)$ can be derived (in general) by solving the Volterra equation (3.8). Since it is well known ${ }^{(6)}$ how $\kappa(x, y, t)$ takes into account the bound states, we have here a good alternative to the formula (3.4). In addition, the motion invariant $s^{-}(k, t)$ can be managed in the same way. First notice that it follows from (3.5) that $e^{-i k x} f^{+}(k, x, t)$ goes to 1 as $\operatorname{Im} k \rightarrow+\infty$, and using this result in $s^{-}(k, t)$ shows an obvious motion invariant of Equation (1.4), $\int_{-\infty}^{+\infty} a(x, t) d x$. If we insert (3.5) in (3.6) for $s^{-}$, we get the sum of this invariant and

$$
J(k)=\int_{0}^{\infty} e^{i k z} d z \int_{-\infty}^{+\infty} \kappa(x, x+z, t) a(x, t) d x
$$

which shows that for any $z>0, \int_{-\infty}^{+\infty} \kappa(x, x+z, t) a(x, t) d x$ is a time invariant.

\section{Boundary condition on $x \geqslant 0, t \geqslant 0, x t=0$.}

We are given $a(x, 0)$ for $x \geqslant 0, a(0, t)$ for $t \geqslant 0$, of course continuous at $(0,0)$, with the derivatives implied by our assumptions of section 2 , all these functions going to zero rapidly enough (for scattering problems), as $x \rightarrow+\infty$. We wish to determine $a(x, t)$ for any finite $t>0, x \in \mathbb{R}^{+}$, going to zero as $x \rightarrow+\infty$, and solution of (1.4). Hence, we have to enforce $\delta^{2} / \Phi=0$ in the quarter-plane, implying therefore Eq. (3.1) and, by integration

$$
\frac{\partial}{\partial t} \int_{0}^{\infty} \widetilde{E}^{ \pm} \tau A G d x=\left[\widetilde{E}^{ \pm} \tau B G\right]_{x=0}^{x=\infty}
$$

In section 3 , we had used $G=G^{+}(k, x, t)$ so as to emphasize the similarities with the classical Inverse Scattering Transform. Here we shall write

$$
G=G^{+}(-k, x, t)=\left(\begin{array}{c}
g(-k, x, t) \\
g^{\prime}(-k, x, t)
\end{array}\right)
$$

were we drop the + index because we shall never use the minus one, negative $x$ being out of our domain. We do so in order to follow more closely the analysis previously written for $\mathrm{KdV}$. However, notice that $G$ here was previously called $F$, and $h, \widetilde{h}$, to be defined below, were called $g, \widetilde{g}$. We set 


$$
\begin{aligned}
& h(k, x, t)=\exp \left[i\left(k x+k^{3} t\right)\right] g(k, x, t) \\
& \widetilde{h}(k, x, t)=\exp \left[-i\left(k x+k^{3} t\right)\right] g(k, x, t)
\end{aligned}
$$

Notice that $g(k, x, t)$ is equal to $e^{i k^{3} t} f^{+}(k, x, t)$, where $f^{+}(k, x, t)$ is still given at $x>0$ by (3.5), so that $h$ and $\widetilde{h}$ are holomorphic in $\operatorname{Im} k \geqslant 0$.

Thanks to $(2.3), g(k, x, t)$ is a solution of an $x$-second order equation, the Schrödinger one, with potential $V(x, t)$, and its time derivative is also a linear combination of $g$ and $g^{\prime}$, with parameters $V_{0}, V_{1}, V_{2}$. As in our previous paper we get from these equations first the following ones :

$$
\begin{aligned}
& h^{\prime \prime}=2 i k h^{\prime}+V h \quad ; \quad \widetilde{h}^{\prime \prime}=-2 i k \widetilde{h}^{\prime}+V \widetilde{h} \\
& \frac{\partial h}{\partial t}=\left(k^{2}+V_{0}\right) h^{\prime}+\left(V_{1}-i k V_{0}\right) h \\
& \frac{\partial h^{\prime}}{\partial t}=\left(2 i k^{3}+i k V_{0}-V_{1}\right) h^{\prime}+\left(2 k^{2} V_{0}+2 i k V_{1}+V_{2}\right) h
\end{aligned}
$$

and for $\widetilde{h}$ those obtained from (4.5), (4.6), (4.7) if we replace $i$ by $-i$. In addition, a little bit of algebra yields :

$$
\begin{aligned}
& h(-k) h^{\prime}\left(k^{\prime}\right)-h\left(k^{\prime}\right) h^{\prime}(-k)=-\frac{\partial}{\partial x}\left\{\frac{k^{\prime}+k}{k^{\prime}-k} h(-k) h\left(k^{\prime}\right)\right. \\
& \left.+\frac{i}{k^{\prime}-k}\left[h(-k) h^{\prime}\left(k^{\prime}\right)-h\left(k^{\prime}\right) h^{\prime}(-k)\right]\right\} \\
& \widetilde{h}(-k) h^{\prime}\left(k^{\prime}\right)-h\left(k^{\prime}\right) \widetilde{h}^{\prime}(-k)=-\frac{\partial}{\partial x}\left\{\frac{k^{\prime}-k}{k^{\prime}+k} \widetilde{h}(-k) h\left(k^{\prime}\right)\right. \\
& \left.+\frac{i}{k^{\prime}+k}\left[\widetilde{h}(-k) h^{\prime}\left(k^{\prime}\right)-h\left(k^{\prime}\right) \widetilde{h}^{\prime}(-k)\right]\right\}
\end{aligned}
$$

Deriving $h^{\prime \prime \prime}$ from (4.5) and adding the result to (4.6), we obtain

$$
\frac{\partial h}{\partial t}+\frac{1}{4} h^{\prime \prime \prime}=\frac{3}{4} V h^{\prime}
$$


whose derivative and (2.12) show that $h^{\prime}$ is a solution of (1.4), so that, provided there is no convergence problem, it is sound to seek a solution of (1.4) of the form

$$
a(x, t)=-2 \int d \mu\left(k^{\prime}\right) h^{\prime}\left(k^{\prime}, x, t\right)
$$

As in our previous paper we decide that

$$
\text { Suppt }[\mathrm{d} \mu]=\mathbb{R}+\Sigma+\Lambda
$$

where $\Sigma$ is a finite set of points, for example $k=i \sigma_{1}, i \sigma_{2}, \cdots i \sigma_{N},\left(\sigma_{n}>0\right.$, and $\Lambda$ is an open contour made of the ray $k=|k| e^{i \pi / 3}$ oriented from $\infty$ to 0 and the ray $k=|k| e^{2 i \pi / 3}$ oriented from 0 to $\infty$. We set $d \omega(k)$ equal to $\xi(k) d k$ along $\mathbb{R}$, made of Dirac measures, for example $\Sigma_{n} \rho_{n} \delta\left(k-i \sigma_{n}\right)$ on $\Sigma$, and $\tau(k) d k$ along $\Lambda$. $\Lambda$, which we called the ternary support, was introduced (originally by Fokas), for the following property : let $\bar{\Lambda}$ be the domain embraced by $\Lambda$, (i.e. phase of $k$ between $\frac{\pi}{3}$ and $\frac{2 \pi}{3}$ ). It is clear that if $\tau(k)$ is holomorphic in $\bar{\Lambda}$ where $\left|k^{-1} \tau(k)\right|$ remains bounded at $\infty$,

$$
a^{T}(x, 0)=-2 \int_{\Lambda} h^{\prime}\left(k^{\prime}, x, 0\right) \tau\left(k^{\prime}\right) d k^{\prime}=0
$$

for any $x \geqslant 0$. Hence, using it as a working assumption on $\tau$ reduces the $t=0$ boundary condition of $a(x, t)$ to a boundary condition of $a^{S}(x, t)$ :

$$
-\frac{1}{2} a^{S}(x, 0)=\int_{\mathbb{R}} \xi\left(k^{\prime}\right) h^{\prime}\left(k^{\prime}, x, 0\right) d k^{\prime}+\sum_{n=1}^{N} \rho_{n} h^{\prime}\left(i \sigma_{n}, x, 0\right)
$$

Let us now successively enforce the conditions on $a(x, t)$

Consistency conditions

The conditions (4.1) are explicitly

$$
\begin{aligned}
& \dot{I}_{1}=: \frac{\partial}{\partial t} \int_{0}^{\infty} d y g(-k, y, t) e(-k, y, t) a(y, t) \\
& =g^{\prime}(-k, 0, t) e(-k, 0, t)\left[a_{1}(0, t)-i k a_{0}(0, t)\right] \\
& -g(-k, 0, t) e(-k, 0, t)\left[a_{2}(0, t)+i k a_{1}(0, t)+k^{2} a_{0}(0, t)\right] \\
& \dot{I}_{2}=: \frac{\partial}{\partial t} \int_{0}^{\infty} d y g(-k, y, t) e(k, y, t) a(y, t)
\end{aligned}
$$




$$
\begin{aligned}
& =g^{\prime}(-k, 0, t) e(k, 0, t)\left[a_{1}(0, t)+i k a_{0}(0, t)\right] \\
& -g(-k, 0, t) e(k, 0, t)\left[a_{2}(0, t)-i k a_{1}(0, t)+k^{2} a_{0}(0, t)\right]
\end{aligned}
$$

If we insert (4.11) in the right hand side of (4.15) and (4.16), and use the formulas (4.8) and (4.9), we obtain after elementary but lengthy calculations

$$
\begin{aligned}
& \dot{I}_{1}=-e^{-i k^{3} t}\left\{g^{\prime}(-k, 0, t)[\alpha+i k C]-g(-k, 0, t)\left[\beta-\gamma+i k \alpha-k^{2} C\right]\right\} \\
& \dot{I}_{2}=-e^{i k^{3} t}\left\{g^{\prime}(-k, 0, t)[\alpha-i k C]-g(-k, 0, t)\left[\beta-\gamma-\left(i k \alpha-k^{2} C\right)\right]\right\}
\end{aligned}
$$

where $\alpha, \beta, \gamma$ and $C$ are functions of $t$ only, herafter defined :

$$
\begin{aligned}
& C(t)=-\int d \mu(k) h^{\prime}(k, 0, t) \\
& \alpha(t)=-\int d \mu(k)\left[V_{0}(0, t) h(k, 0, t)+i k h^{\prime}(k, 0, t)\right] \\
& \beta(t)=\int d \mu(k)\left[V_{1}(0, t)+i k V_{0}(0, t)\right] h(k, 0, t) \\
& \gamma(t)=-\int d \mu(k)\left[\frac{1}{2} V_{0}(0, t)+k^{2}\right] h^{\prime}(k, 0, t)
\end{aligned}
$$

Reminding that $e(k, 0, t)$ is $e^{i k^{3} t}$, we can compare the right-hand sides of (4.15) and (4.17), then those of (4.16) and (4.18), and identify the coefficients of $g(k, \cdots), g^{\prime}(k, \cdots)$ and the powers of $k$. The cancelling of coefficients ensures the consistency. More precisely, setting

$$
P(k)=\alpha+a_{1}-i k\left(C-a_{0}\right)
$$

where $a_{1}$ and $a_{0}$ stand for $a_{1}(0, t)$ and $a_{0}(0, t)$, we get

$$
\begin{aligned}
& a_{0}(0, t)=C(t) \\
& a_{1}(0, t)=-\alpha(t)
\end{aligned}
$$




$$
a_{2}(0, t)=-\beta(t)+\gamma(t)
$$

Hence, because of the consistency relations, if $d \mu(k)$ can be identified from the values of $a(x, 0)$ and one of the functions $a_{0}(0, t), a_{1}(0, t), a_{2}(0, t)$, the two other ones are determined by the relations (4.24), (4.25), (4.26).

Boundary conditions on $x \geqslant 0, t=0$

Going back to (4.14), we work with the integrated boundary condition

$$
A(x)=\frac{1}{2} \int_{x}^{\infty} a(y, 0) d y
$$

which fits (4.14) if $h$ is replaced by its value, derived from (3.5) :

$$
\begin{aligned}
& h(k, x, t)=e^{2 i k^{3} t}\left[e^{2 i k x}+\int_{x}^{\infty} \kappa(x, y, t) e^{i k(x+y)} d y\right] \\
& \text { and } A(x)=\widetilde{A}(x)+\int_{x}^{\infty} \kappa(x, y, 0) \widetilde{A}\left(\frac{x+y}{2}\right) d y \\
& \text { where } \widetilde{A}(x)=\int_{\mathbb{R}} \bar{\xi}\left(k^{\prime}\right) e^{2 i k^{\prime} x} d k^{\prime}+\sum_{n=1}^{N} \rho_{n} e^{-2 \sigma_{n} x}
\end{aligned}
$$

The equation (4.29) is a regular linear Volterra equation, with integrable kernel and its unique solution yields $\widetilde{A}(x)$ from $A(x)$. Except for a remark later we study only the case where the support $\Sigma$ is entirely in $\operatorname{Im} k>0$. Then it can be suppressed in (4.12), (4.14) and (4.30) provided $\bar{\xi}(k)$ is replaced by $\xi(k)$, obtained by adding to $\bar{\xi}(k)$ a convenient function (which is $\left.(\pi i)^{-1} \Sigma_{n} \rho_{n}\left(k-i \sigma_{n}\right)^{-1}\right)$ in the given example. The problem of fitting the boundary condition at zero $t$ reduces to that of deriving $\xi(k)$ from a knowledge of $\widetilde{A}$ on $x>0$ only, and

$$
\widetilde{A}(x)=\int_{\mathbb{R}} \xi(k) e^{2 i k x} d k
$$

Its general solution is

$$
\xi(k)=\pi^{-1} \int_{0}^{\infty} \widetilde{A}(x) e^{-2 i k x} d x+\int_{-\infty}^{0} \widetilde{\eta}(x) e^{-2 i k x} d x
$$

where $\widetilde{\eta}(x)$ is arbitrary in $L^{1}\left(\mathbb{R}^{-}\right)$and therefore the second term $\eta(k)$ in (4.32) is holomorphic and bounded in $\operatorname{Im} k>0$, whereas the first one, $\xi_{0}(k)$, is so in $\operatorname{Im} k<0$. We can suppress $\eta(k)$ in (4.32) because, according to (4.28), its contribution to (4.11) : 


$$
I(\eta)=\int_{\mathbb{R}} \eta(k) h^{\prime}(k, x, t) d k
$$

is the integral of a function that is holomorphic and bounded in the domains $0 \leqslant \operatorname{Arg} k \leqslant$ $\frac{\pi}{3}$ and $\frac{2 \pi}{3} \leqslant \operatorname{Arg} k \leqslant \pi$, where $e^{2 i k^{3} t}$ collapse at $\infty$, so that

$$
I(\eta)=-\int_{\Lambda} \eta(k) h^{\prime}(k, . x, . t) d k
$$

Hence suppressing $\eta$ in (4.32) is equivalent to adding it to $\tau(\mathrm{k})$, which is still to be determined, and in the following we seek $a(x, t)$ as

$$
-\frac{1}{2} a(x, t)=\int_{\mathbb{R}} \xi_{0}(k) h^{\prime}(k, x, t) d k+\int_{\Lambda} \tau(k) h^{\prime}(k, x, t) d k
$$

where $\xi_{0}$ has now been determined, and $\tau(k)$ is to be determined from the $t$-axis boundary condition and should satisfy the "working assumption on $\tau$ ".

Boundary condition on $t>0, x=0$

Because the equation (1.4) is linear, the consistency relations hold as well for

$$
a^{T}(x, t)=a(x, t)-a^{S}(x, t)=-2 \int_{\Lambda} \tau(k) h^{\prime}(k, x, t) d k
$$

and $a^{T}(0, t)$ is readily obtained from $a(0, t)$ and the calculated $a^{S}(0, t)$. Hence we must solve

$$
-\frac{1}{2} a^{T}(0, t)=\int_{\Lambda} \tau(k) h^{\prime}(k, 0, t) d k
$$

where

$$
h^{\prime}(k, 0, t)=e^{2 i k^{3} t}\left\{2 i k+\int_{0}^{\infty}\left[\left(\frac{\partial}{\partial x}-\frac{\partial}{\partial y}\right) \kappa(x, y, t)\right]_{x=0} e^{i k y} d y-2 \kappa(0,0, t)\right\}
$$

We seek $\tau(k)$ in the form

$$
\tau(k)=k \int_{0}^{\infty} \widetilde{\tau}(u) e^{-2 i k^{3} u} d u
$$

which enforces the working assumption on $\tau$ if $\widetilde{\tau}$ belongs to $L^{1}\left(\mathbb{R}^{+}\right)$. Introducing (4.39) in (4.37) yields the integral equation for $\widetilde{\tau}$ :

$$
-\frac{1}{2} a^{T}(0, t)=\frac{2 i \pi}{3} \widetilde{\tau}(t)+\int_{0}^{t} S(t, u) \widetilde{\tau}(u) d u
$$


where

$$
\begin{aligned}
& S(t, u)=\int_{\Lambda} e^{2 i k^{3}(t-u)} k d k\{-2 \kappa(0,0, t) \\
& \left.+\int_{0}^{\infty} d y e^{i k y}\left[\left(\frac{\partial}{\partial x}-\frac{\partial}{\partial y}\right) \kappa(x, y, t)\right]_{x=0}\right\}
\end{aligned}
$$

The integral on $k$ in (4.41) vanishes for $u>t$ because then $k e^{2 i k^{3}(t-u)}$ collapses at $\infty$ in $\bar{\Lambda}$ and it is multiplied by a function which is holomorphic and bounded in $\bar{\Lambda}$. For $t>u$, the first term of (4.41) can be calculated exactly, giving say,

$$
S_{0}(t, u)=4 i \sin \left(\frac{\pi}{3}\right) \kappa(0,0, t)(t-u)^{-2 / 3}
$$

The second term, say, $S_{1}(t, u)$, involves integrals as

$$
I=\int_{0}^{\infty} q d q e^{i f(q)}
$$

where

$$
f(q)=i\left[2 q^{3}(t-u)+q y \cos \frac{\pi}{3}\right]-q y \sin \frac{\pi}{3}
$$

or its conjugate. It is clear that for $t-q$ and/or $y>0, f^{\prime}(q)$ is bounded away from zero. Integrating by part we easily get

$$
|I| \leqslant \int_{0}^{\infty} e^{-q y \sin \pi / 3}\left[36 q^{4}(t-u)^{2}+y^{2} \sin ^{2} \frac{\pi}{3}\right] d q
$$

from which bounds by $y^{-1}$ or $y^{-1 / 2}(t-u)^{-1 / 2}$ are easily derived. After performing the integration on $y$ we show readily that $|t-u|^{1 / 2}\left|S_{1}(t, u)\right|$ is bounded at finite $t>0$, and gathering this result with (4.42), we see that the kernel of (4.40) is only weakly singular and that the Volterra equation (4.40) has a unique solution, finite as long as $t$ is finite. The behavior of $\widetilde{\tau}(t)$ at $\infty$ depends on that of $a^{T}(0, t)$, and also of that of the functions $\kappa$ related to $V$. If $V$ decreases rapidly enough, and $a^{T}(0, t)$ belongs to $L^{1}\left(\mathbb{R}^{+}\right), \widetilde{\tau}(t)$ does it also, but apart this remark which gives already a wide class of possible boundary conditions, we shall not study it more.

\section{$5 \quad$ On motion invariants}

We have assumed that $V(x, t)$, which is a parameter in Eq. (1.4), is a solution of KdV, with conditions (in section 3), that allow classical inverse scattering. Hence its Jost solution 
$f^{+}(k, x, t)$, (see Eq. 3.5), is related to $f^{-}(k$,$) and f^{-}(-k$,$) by a transmission coefficient$ $T(k)$ and a reflection coefficient $R^{-}(k) e^{-2 i k^{3} t}$, where $T$ and $R^{-}$are $\mathrm{KdV}$ motion invariants

$$
f^{+}(k, x, t) \sim \begin{cases}e^{i k x} & x \rightarrow \infty \\ \frac{e^{i k x}}{T(k)}+\frac{R^{-}(k) e^{-2 i k^{3} t-i k x}}{T(k)} & x \rightarrow-\infty\end{cases}
$$

It is possible to relate the motion invariants of Equation (1.4), namely $s^{-}(k, t)$ and $e^{2 i k^{3} t} s^{+}(k, t)$, to $T$ and $R^{-}$. In the particular case where the data, $a(x, 0)$ itself has been chosen equal to $V(x, 0)$, the solution of Eq. (1.4) is $V(x, t)$. Indeed, using Eqs. (4.3), (4.4), (4.5), where $g$ is equal to $e^{i k^{3} t} f^{+}(k, x, t)$ and (5.1), we readily obtain :

$$
s^{+}(k, t) e^{2 i k^{3} t}=2 i k \frac{R^{-}(k)}{T(k)} \quad ; \quad s^{-}(k, t)=1-\frac{1}{T(k)}
$$

A much more general example is obtained by introducing a more general data :

$$
a(x, 0)=\pi \int_{\mathbb{R}} \alpha(\ell) h^{\prime}(\ell, x, 0) d \ell
$$

where $\alpha(\ell)$ and derivative belong to $L^{1}(\mathbb{R})$ and is such that $a(x, 0)$ and $s^{ \pm}(k, 0)$ belong to $L^{1}(\mathbb{R})$ but otherwise can be chosen arbitrarily (in particular it may be any function of the space $S$ which is commonly used for defining tempered distributions). Thanks to (4.10), we can write down :

$$
a(x, t)=\pi \int_{\mathbb{R}} \alpha(\ell) h^{\prime}(\ell, x, t) d \ell
$$

Now let us calculate

$$
s^{+}(k, t) e^{2 i k^{3} t}=\lim _{X \rightarrow \infty} \int_{-X}^{X} a(x, t) h(k, x, t) d x
$$

It is most convenient to achieve this derivation in the sense of tempered distributions by first calculating for any $\varphi \in \delta$ :

$$
\left\langle s^{+} e, \varphi\right\rangle=\int_{-\infty}^{+\infty} \varphi(k) s^{+}(k, t) e^{2 i k^{3} t} d k
$$

Inserting (5.4) into (5.5), (5.5) into (5.6), and using (5.1), the oscillating terms can be managed and we get :

$$
s^{+}(k, t) e^{2 i k^{3} t}=2 i \pi k \alpha(-k)-\frac{R^{-}(k)}{T(k)} V_{P} \int_{-\infty}^{+\infty} \alpha(\ell) \frac{R^{-}(\ell)}{T(\ell)} \frac{\ell}{k+\ell} d \ell
$$

( $V_{P}$ is the Cauchy Principal Value). Similar results hold for $s^{-}(k, t)$. 


\section{Conclusion}

The initial value problem and the quarter-plane problem can generally be solved for the equation (1.4). This has already suggested to the author a first attempt to solve the quarter-plane problem for KdV but with little success. On the other hand, the solution of the equation (1.4), which mixes harmonic analysis and integral equations is beautiful. It uses specifically the spectrum of a Schrödinger operator (that of $V$ ) but not "that" of $a$, and the equation (1.4) is linear. Thus it leads us to ask Francesco Calogero the following question "would you say this equation is $C$-integrable or $S$-integrable" ? Notice finally that it is possible to apply the method to other linear p.d.e. "neighbouring" nonlinear integrable ones

\section{Acknowledgements}

We thank Professors M. Boiti and F. Pempinelli for their reading of the manuscript and valuable comments.

\section{References}

[1] F. Calogero and A. Degasperis. "Spectral transfom and solitons" North Holland, Amsterdam - New York - Oxford 1982.

[2] P.C. Sabatier, JMP 44, 3216-3225 (2003). Please correct the sign of (5.28) according to that in the present formula (4.26).

[3] A.S. Fokas. Selecta Math. New Ser. 4, 31 - (1998) ; Proc. R. Soc. London. Ser. A 457, 371 - (2001) ; IMAJ. Appl. Math. 67, 559 - (2002).

[4] P.C. Sabatier Inverse Problems 18, 611-630 (2002) ; "A workmate for KdV", p. 118-124 in "Nonlinear Physics Theory and Experiment II" M.J. Ablowitz, M. Boiti, F. Pempinelli, B. Prinari eds World Scientific New Jersey - London (2003).

[5] This formula was given by E.K. Khristov in a Dubna preprint of 1980 (P.5-80-381), probably published in an ukranian journal on differential equations. In our book (following reference) we reproduced it page 348 [formulas XVII 3.21] but with an error : $k / T(k) \widetilde{T}(k)$ should be replaced by its inverse, as it has been done in the present paper after checking again the formula.

[6] K. Chadan, P.C. Sabatier. "Inverse Problems in Quantum Scattering Theory" 2nd edition Springer. New York - Berlin - Heidelberg (1989). 